(ROS) are known to play a central role in the tissue damage caused by ischaemia-reperfusion injury (IRI), yet the use of antioxidant supplements in large scale clinical trials has been shown to have no beneficial effect and indeed some studies have suggested that the addition of exogenous ROS may decrease infarct size. This suggests that mitochondria may exhibit a biphasic response to ROS, termed mitohormesis, characterised by beneficial effects at low doses and detrimental effects at high doses. To investigate this phenomenon, a tool is required to precisely titrate mitochondrial ROS. MitoParaquat is a novel mitochondria-targeted molecule that redox cycles at complex I to produce superoxide, closely mimicking the production of superoxide as the proximal ROS species in vivo. Here it is used to investigate the role of ROS in protection against acute myocardial IRI.

Methods Male C57BL6/J mice aged 8-10 weeks were administered MitoParaquat or vehicle only control by intravenous injection $15 \mathrm{~min}$ before the induction of $30 \mathrm{~min}$ myocardial ischaemia by ligation of the left anterior descending coronary artery. After 2 hours of reperfusion, infarct size was determined by tripheyltetrazolium chloride staining.

Results MitoParaquat decreased infarct size relative to vehicle only control $(42.3 \pm 4.3 \%)$ at doses of $1 \mathrm{nmol}(30.0 \pm 4.0 \%)$, $100 \mathrm{pmol}(22.7 \pm 2.3 \%)$, and $10 \mathrm{pmol}(24.2 \pm 2.0 \%)$. At 1 pmol, no significant difference from vehicle only control was observed $(41.5 \pm 8.6 \%)$, and at $5 \mathrm{nmol}$ it was found to be lethal. There was no significant difference in the area at risk between any groups.

Conclusions First and foremost, the generation of low doses of exogenous ROS by MitoParaquat is shown to be protective against acute myocardial IRI in vivo. MitoParaquat is shown to exhibit a hormetic dose response curve, with protection conferred only in an intermediate dose range with high doses found to be lethal and infarcts from low dose not significantly different from control. Further work is required to determine the mechanism by which this cardioprotection occurs.

\section{EVALUATION OF ACUTE CARDIAC DAMAGE IN A PORCINE MODEL OF DEFIBRILLATION}

${ }^{1}$ Fiona Rogan*, ${ }^{2}$ Rebecca Funston, ${ }^{1}$ Brian Meenan, ${ }^{1}$ George Burke. ${ }^{1}$ Ulster University; ${ }^{2}$ Heartsine Technologies

\subsection{6/heartjnl-2017-311726.207}

Introduction Transthoracic defibrillation is performed to correct life threatening cardiac arrhythmias of the heart, i.e. ventricular fibrillation (VF) and ventricular tachycardia (VT). These are due to chaotic electrical excitation of the heart chambers and loss of coordinated contraction of myocytes that could induce cardiac arrest. Resuscitation guidelines recommend defined defibrillation energies for patient recovery, research indicates that these techniques may cause myocardial damage. However, there is limited information regarding the defibrillation potentially damaging effects on the tissues of the heart at structural and genomic levels. This study investigates the extent of myocardial injury associated with the use of increasing energies $(75 \mathrm{~J}, 150 \mathrm{~J}, 200 \mathrm{~J}$ and $360 \mathrm{~J}$ ) of defibrillation in a porcine model of cardiac arrest using genomic, histological and ultrastructural analysis techniques.

Methodology General anaesthesia was induced in swine models ( $\tilde{\mathrm{A}} \notin \hat{\mathrm{a}} €{ }^{\circ} \ddot{\mathrm{E}} \dagger 10-40 \mathrm{~kg}$ ) in accordance with The Home Office guidelines. VF was induced and defibrillation administered, each animal receiving 20 shocks at the defibrillation energy protocol at $3 \mathrm{~min}$ intervals followed by 6 hour recovery period. Upon completion, animals were humanely euthanised. Cardiac tissues were excised and processed for genomic, histological and ultrastructural analyses and examination.Results: Haemodynamic results demonstrated ROSC occurred in all pigs. Troponin I levels were elevated 3-4 hours after the completion of defibrillation protocol. Gross pathological examination demonstrated no unusual changes. Histological and SEM analysis indicate defibrillation causes changes to the porcine cardiac tissue as evidenced by instances of hypereosinophilia, increased collagen-I deposition and areas of multifocal acute subendocardial, epicardial and myocardial necrosis. qPCR analysis indicates defibrillation induces genomic changes, there was an upregulation in the mRNA expression of structural and inflammation related genes such as Collagen-I, IL-6/18 and MCP1. Hydroxyproline analysis and SEM imaging also illustrated minor changes in collagen content and structural appearance of the tissue, further supported with Image J colour hue analysis.

Discussion The current paradox is cardiac defibrillation depends on the successful selection of energy to generate sufficient current flow through the heart to achieve defibrillation, whilst causing minimal injury to the heart. At this acute timeframe (post protocol), the animal models illustrated biological effects from repeated defibrillation upon cardiac tissues at a structural and genomic level and suggests there is a cardioprotective measure taken by the cardiomyocytes due to electrical overstimulation. In conclusion, these results show repeated defibrillation at increasing energies produces immediate changes to the functional myocardium at a genomic, microscopic and ultrastructural level.

\section{SYNDECAN-4/FGF-2/PKCA SIGNALLING REGULATES VASCULAR SMOOTH MUSCLE CELL CALCIFICATION VIA CROSS-TALK WITH TGFB}

${ }^{1}$ Samantha Borland*, 'Shona Borland, ${ }^{2}$ Mark Morgan, ${ }^{3}$ Sheila Francis, ${ }^{4}$ Catherine Merry, ${ }^{1}$ Ann Canfield. ${ }^{1}$ University of Manchester; ${ }^{2}$ University of Liverpool; ${ }^{3}$ University of Sheffield; ${ }^{4}$ University of Nottingham

\subsection{6/heartjin-2017-311726.208}

Vascular smooth muscle cells (VSMCs) were induced to mineralise with $\beta$-glycerophosphate ( $\beta$-GP). Controls were cultured without $\beta$-GP. FGF-2 mRNA $(\sim 40$-fold increase, $\mathrm{P}<0.001)$ and protein $(\sim 2$-fold increase, $\mathrm{P}<0.05)$ expression are significantly increased in mineralising VSMCs. FGF-2 also localises to sites of calcification within human atherosclerotic plaques. The expression of syndecan-4, a heparan sulphate proteoglycan which acts as a co-receptor for FGF-2 signalling, is also increased in mineralising VSMCs $(\sim 5$-fold, $\mathrm{P}<0.001)$ and co-localizes with FGF-2 in human calcified atherosclerotic plaques. Exogenous FGF-2 inhibits VSMC mineralisation $(\mathrm{P}<0.001)$ and this inhibition is reduced when syndecan-4 expression is knocked-down using siRNA.

Biochemical inhibition of FGFR signalling using a pan FGFR inhibitor (BGJ398) increases transforming growth factor- $\beta 1$ (TGFß1)-induced Smad2 phosphorylation in VSMCs. As TGF $\beta 1$ increases mineral deposition by VSMCs $(\sim 2$-fold, $\mathrm{P}<0.01)$, the relationship between FGF and TGF $\beta$ signalling in VSMC mineralisation was investigated. Inhibiting FGFR signalling using BGJ398 or knocking-down syndecan-4 expression in VSMCs 
using siRNA increases VSMC mineralisation (both $\mathrm{P}<0.001$ ). These increases are prevented by inhibiting $\mathrm{TGFI}^{2}$ signalling with SB431542, suggesting cross-talk between FGF-2 and TGFI' ${ }^{2}$ signalling is crucial for the regulation of VSMC mineralisation. Syndecan-4 can also regulate FGF-2 signalling via protein kinase $\mathrm{C} \alpha(\mathrm{PKC} \alpha)$ activation. Biochemical inhibition of PKC $\alpha$ activity using G6976, or knocking-down PKC $\alpha$ expression increases VSMC mineralisation (both $\mathrm{P}<0.05$ ); this increase is also prevented with SB431542. Finally, the ability of FGF-2 to inhibit VSMC mineralisation is reduced when PKC $\alpha$ expression is knocked-down.

In conclusion, our study has identified that syndecan-4/FGF-2 signalling is up-regulated in mineralising VSMCs to reduce $\mathrm{TGF}^{2}$ signalling and minimise further calcification. Syndecan-4 regulates FGF-2 signalling to prevent excessive mineralisation by (a) acting as a co-receptor for FGF-2 and inducing downstream signalling via FGFR and (b) interaction with PKo. The syndecan4/FGF-2/TGF $\alpha$ signalling axis could therefore represent a new therapeutic target for vascular calcification.

\section{CHARACTERISING FUNCTIONAL HETEROGENEITY IN HUMAN EPICARDIUM}

${ }^{1}$ Sophie McManus*, 'Laure Gambardella, ${ }^{1}$ Victoria Moignard, 'Dharini lyer, ${ }^{1}$ Bertie Gottgens, ${ }^{2}$ Nicolas Le Novere, 'Helle Jorgensen, 'Sanjay Sinha. 'University of Cambridge; ${ }^{2}$ Babraham Institute

\subsection{6/heartjnl-2017-311726.209}

Introduction The epicardium is a heterogeneous cell layer covering the mammalian heart. During embryogenesis, the epicardial lineage is essential to heart and vascular development, yielding cardiac fibroblasts and coronary vascular smooth muscle cells. The epicardium also has a trophic effect on developing cardiomyocytes. It is quiescent in adulthood but reactivates post-injury to a limited degree to yield cardiac fibroblasts, which allows fast healing, yet also causes fibrosis. Epicardial functional heterogeneity remains incompletely characterised.

Methods The Sinha group has derived a robust model of human epicardium (hpsc-epi) from human pluripotent stem cells; this was used for single-cell RNA sequencing (scRNAseq). Immunohistochemistry and immunocytochemistry were used to validate scRNA-seq results in primary human foetal tissue. The candidate gene BNC1 has been investigated by siRNA-mediated knockdown studies in vitro.

Results Single-cell RNA-seq identified two main epicardial subpopulations in hpsc-epi: WT $1^{\text {high }} / \mathrm{BNC} 1^{\text {high }} / \mathrm{TCF} 21^{\text {low }}$ and WT $1^{\text {low }} / \mathrm{BNC} 1^{\text {low }} / \mathrm{TCF} 21^{\text {high }}$. Here we show validation of our scRNA-seq data in human foetal epicardium by immunohistochemistry in cryosections and human foetal epicardial explants, confirming our hpsc-epi model is representative of the in vivo situation. We show preliminary data from siRNA-mediated knockdown of BNC1, which indicate this gene may play a role in epicardial function, possibly in regulating cell migration in a model of epithelial-to-mesenchymal transition.

Implications Improved understanding of developmental epicardial regulation could pave the way towards harnessing epicardial potential in prospective strategies to aid revascularisation and regeneration of the injured heart.

\section{SUPPRESSOR OF CYTOKINE SIGNALLING 3 (SOCS3) INTERACTION WITH CAVIN-1 LINKS SOCS3 FUNCTION AND CAVIN-1 STABILITY}

${ }^{1}$ Jamie Williams*, ${ }^{2}$ Nasser Alotaiq, ${ }^{1}$ Tim Palmer. ${ }^{1}$ School of Pharmacy, University of Bradford; ${ }^{2}$ Institute of Cardiovascular and Medical Sciences, University of Glasgow

\subsection{6/heartjnl-2017-311726.210}

Caveolae are lipid raft microdomains essential for the compartmentalisation and regulation of several signalling pathways e.g. JAK/STAT signalling. Disruption of caveolae is a significant factor in multiple disorders including muscular dystrophy, cardiovascular disease, and cancer. Central to caveolae stability is cavin-1 which couples caveolae to the microtubule network to prevent degradation of a key structural element i.e. caveolin-1, and caveolae disassembly. Via an unbiased quantitative proteomics screen, we have identified SOCS3, a negative regulator of JAK/STAT signalling, as a novel cavin-1 interactor.

SOCS3-cavin-1 interactions were characterised by immunoprecipitation assays and probing overlapping peptide arrays. SOCS3 bound to multiple regions within cavin-1, while a PEST motif within the C-terminal region of the SOCS3 $\mathrm{SH} 2$ domain was required for interaction with cavin-1 independently of its capacity to bind phospho-tyrosine. Biochemical analysis and confocal imaging also demonstrated that SOCS3 localisation within lipid raft microdomains and at the plasma membrane required cavin-1. Interestingly, SOCS3 does not ubiquitinate cavin-1 but instead supports cavin-1/caveolae stability. Moreover, genetic deletion of cavin-1 results in the loss of SOCS3-mediatied inhibition of cytokine signalling. Importantly, while the inhibitory function of SOCS3 relies on its induction, caveolae stabilisation occurs at basal SOCS3 expression levels. Thus, transmission electron microscopy demonstrated that SOCS3 knock-out endothelial cells show reduced levels of caveolae.

Our data suggest a novel role for SOCS3 in regulating caveolae assembly while cavin-1, acting as a scaffold-protein, might aid SOCS3-dependent regulation of JAK/STAT signalling. This is the first indication of a novel role for SOCS3 in caveola homeostasis and suggests that loss of caveolae represents a novel mechanism by which chronic activation of pro-inflammatory JAK/STAT signalling could be triggered in disease.

\section{INCREASED BETA-AMYLOID PRODUCTION IS ASSOCIATED WITH DIABETES-INDUCED VASCULAR DYSFUNCTION}

Paul Meakin*, Zofia Tuharska, Christopher McCaffery, Faisel Khan, Michael Ashford. University of Dundee

\subsection{6/heartjnl-2017-311726.211}

Introduction $\beta$-amyloid $(A \beta)$ is produced via the cleavage of amyloid precursor protein $\beta$-secretase (BACE1), resulting in the formation of amyloid plaques, a hallmark pathology of Alzheimer's disease (AD). AD, type 2 diabetes, obesity and cardiovascular disease appear intimately linked with endothelial dysfunction, inflammation, insulin resistance and elevated $\mathrm{A} \tilde{\mathrm{A}} f \AA$, levels all common features. Impaired endothelial function is represented by impaired endothelium-dependent, nitricoxide (NO)-mediated relaxation. Tight regulation of vasoactive 